\title{
La formación integral de los estudiantes de Nutrición a partir de extensión universitaria en el medio rural
}

Adriana Cauci

(iD) orcid.org/0000-0003-4846-3015

adrianacauci@gmail.com

\section{Florencia Muniz}

(D) orcid.org/0000-0002-8510-5199

florenciamuniz@gmail.com

\author{
Fernanda Risso \\ (iD) orcid.org/0000-0003-4430-4981 \\ fernandarisso10@gmail.com
}

Escuela de Nutrición de la Universidad

de la República, Uruguay.
Sujetos y relaciones en extensión universitaria / Desafíos de gestión
RECEPCIÓN: 26/06/20

ACEPTACIÓN FINAL: 02/11/20

\section{Resumen}

El equipo interdisciplinario de Nutrición, Veterinaria y Psicología de la Universidad de la República, Uruguay, desarrolla Espacios de Formación Integral desde 2012 en los que participan estudiantes, familias productoras y escuelas rurales. El presente artículo tiene como objetivo rescatar los aportes que estos Espacios vinculados al medio rural realizan a la formación de los Licenciados en Nutrición tomando el período desde su creación hasta 2019. Los Espacios son dispositivos que apuntan a favorecer la articulación de enseñanza, extensión e investigación en el proceso formativo de los estudiantes, promueven el pensamiento crítico y propositivo, y la autonomía de los sujetos involucrados. Para dar cuenta de ello, identificaremos la relevancia de la formación disciplinar para luego consolidarla en el encuentro con otras disciplinas y saberes. La importancia del análisis de la situación alimentaria nutricional desde una visión integral y los marcos conceptuales de seguridad y soberanía alimentaria necesarios para comprender la complejidad de los problemas a nivel poblacional, con acciones integrales para su resolución y el alcance del derecho a la alimentación adecuada.

Palabras clave: extensión universitaria; ruralidad; nutrición.
Comprehensive training of students of Nutrition from university extension in rural areas

\section{Abstract}

The interdisciplinary team of Nutrition, Veterinary Medicine and Psychology of the University of the Republic, Uruguay, develops Integral Training Spaces since 2012 in which students, producer families and rural schools participate. This article aims to rescue the contributions that these Spaces linked to the rural environment make to the training of Nutrition Graduates from the period from their creation to 2019. The Spaces are devices that aim to favor the articulation of teaching, extension and research In the formative process of students, they promote critical and purposeful thinking, and the autonomy of the subjects involved. To account for this, we will identify the relevance of disciplinary training and then consolidate it in the encounter with other disciplines and knowledge. The importance of analyzing the food and nutrition situation from a comprehensive perspective and the conceptual frameworks of food security and sovereignty necessary to understand the complexity of problems at the population level, with comprehensive actions for their resolution and the scope of the right to adequate food.

Keywords: university extension; rurality; nutrition.
Formação integral de alunos do Curso em Nutrição a partir de extensão universitária em áreas rurais

\section{Resumo}

A equipe interdisciplinar de Nutrição, Medicina Veterinária e Psicologia da Universidade da República, Uruguai, desenvolve, desde 2012, Espaços de Formação Integral em que participam alunos, famílias produtoras e escolas rurais. Este artigo objetiva resgatar as contribuições que esses Espaços vinculados ao meio rural trazem para a formação de Graduados em Nutrição desde sua criação até 2019. Os Espaços são dispositivos que visam favorecer a articulação do ensino, extensão e pesquisa. No processo formativo dos alunos, promovem o pensamento crítico e propositivo e a autonomia dos sujeitos envolvidos. Para dar conta disso, vamos identificar a relevância da formação disciplinar e, em seguida, consolidá-la no encontro com outras disciplinas e saberes. A importância de analisar a situação alimentar e nutricional desde uma perspectiva integral e os marcos conceituais de segurança e soberania alimentar necessários para compreender a complexidade dos problemas no nível populacional, com ações abrangentes para sua resolução e o alcance do direito à alimentação adequada.

Palavras-chave: extensão universitária; ruralidade; nutrição. 


\section{Introducción}

El equipo interdisciplinario de Nutrición, Veterinaria y Psicología, con estudiantes y en articulación con las familias productoras y escuelas rurales de la Colonia Alonso Montaño y la Sociedad de Fomento Rural de Pan de Azúcar, desarrolla Espacios de Formación Integral (EFI) desde el año 2012. Estos surgen en el marco de la Segunda Reforma Universitaria y se los define como "ámbitos para la promoción de prácticas integrales en la Universidad, favoreciendo la articulación de enseñanza, extensión e investigación en el proceso formativo de los estudiantes" (Rectorado UdelaR, 2010, pp. 9-10).

En este marco es que la Escuela de Nutrición de la UdelaR inicia un largo proceso de renovación de su plan de estudios, lo cual da origen a un nuevo programa aprobado en 2014 e implementado en 2018. Este se centra en el desarrollo de competencias genéricas y específicas desde una concepción de la ciencia de la nutrición y de la salud que supera la perspectiva biologicista. Se entiende a la nutrición como concepto multidimensional, que contempla también las dimensiones social y ambiental dado que toma en consideración al individuo en su contexto (Manchado et al., 2009) e identifica la multicausalidad de los problemas alimentarios y nutricionales y su vinculación con determinantes políticos, socioeconómicos y medioambientales. Como campo de acción, la nutrición poblacional abreva de distintas áreas, como la nutrición comunitaria en salud pública y la epidemiología nutricional.

El enfoque participativo y el logro del derecho humano a una alimentación adecuada (en adelante lo evocaremos como DAA) en los territorios rurales, y extendiendo la convocatoria más allá del sector salud, incorporando el área agraria a través de la Facultad de Veterinaria, son claves para comprender otros sectores del desarrollo económico y social. La Seguridad Alimentaria y Nutricional y la Soberanía Alimentaria amplían la mirada sobre los aspectos que condicionan la realización del DAA a la luz del Sistema Agro-Alimentario (SAA) actual.

Este trabajo busca rescatar los aportes que los EFI en el medio rural (2012-2019) hacen a la formación de los licenciados en Nutrición. Para alcanzar este objetivo, el equipo se propuso rescatar las prácticas mediante la metodología de sistematización crítica. La misma consta de una reconstrucción de la integración del equipo interdisciplinario y los territorios rurales, así como de la experiencia de trabajo conjunta a partir de la línea de tiempo que intenta recoger el proceso de trabajo y los hitos como los momentos significativos de la experiencia, obtenidos por medio de una nube de palabras.

\section{La integración del equipo interdisciplinario y los territorios rurales}

En 2009-2010 se conformó un equipo de extensión interdisciplinaria integrado por docentes de la Escuela de Nutrición y la Facultad de Psicología y Veterinaria, en el marco del proyecto de extensión universitaria "Prácticas integrales en el medio rural: promoviendo el desarrollo de las comunidades rurales", el cual trabajó con escuelas rurales de Villa del Rosario, Lavalleja, San José y Canelones, y contó con la participación de estudiantes de los tres servicios universitarios.

En el año 2012 el equipo comenzó un nuevo proceso de trabajo con las comunidades rurales de la Colonia Alonso Montaño (AM), en el departamento de San José, y la Sociedad de Fomento Rural (SFR) en Pan de Azúcar, en el departamento de Maldonado (Figura 1). 
Figura 1: Ubicación de los territorios rurales

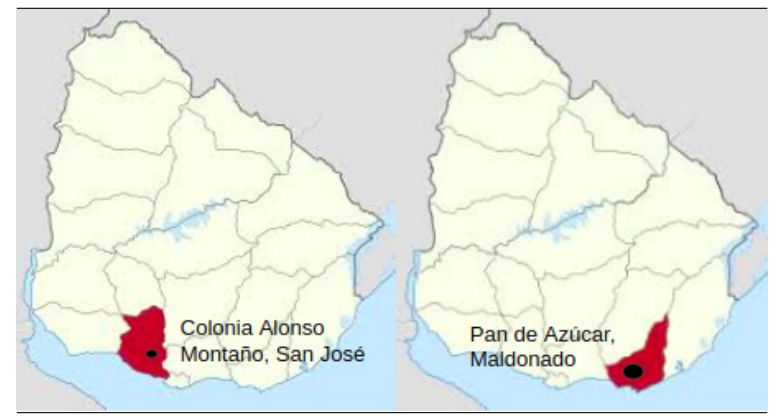

Fuente: elaboración propia.

La SFR ${ }^{1}$ de Pan de Azúcar (PA) nuclea a familias productoras y defiende los intereses de los productores rurales y sus familias en su zona y alrededores. Su territorio de influencia comprende predios en la cuenca de la Laguna del Sauce, en colonias del Instituto Nacional de Colonización (INC) y en los ejes de las Rutas Nacionales 60 y 9, en el departamento de Maldonado. Es una zona de sierras, por lo que los productores familiares asociados presentan varios rubros, entre los que predominan los ganaderos (bovinos y ovinos), aunque también existen agricultores (convencionales y agroecológicos) y apícolas.

La Colonia AM se encuentra ubicada en el departamento San José, en la 6ta. Sección Judicial, al sur de la Ruta 1 desde el Km 61000 hasta el 67 500. Está compuesta por tres inmuebles $\left(443,439\right.$ y 459) y subdividida en 113 fracciones. Hay dos escuelas rurales, $N^{\circ} 92$ y $\mathrm{N}^{\circ} 98$, dos salones comunales y dos clubes deportivos. Casi la mitad de los colonos tiene predios menores de 30 hectáreas; poco más de un tercio de ellos posee entre 30 y 80 hectáreas, y menos de una quinta parte tiene predios mayores de 80 hectáreas. El rubro productivo predominante es la producción láctea, pero el modo particular de inserción en la cadena productiva no es igual para el conjunto de las familias productoras. Existen diferencias tanto por el producto que generan, observándose heterogeneidad en cuanto a la calidad del mismo (leche o elaboración de queso) como también respecto de las instalaciones con que cuentan para la producción y elaboración. Los colonos se agrupan y articulan en diversos colectivos en pro del desarrollo de la Colonia: la comisión de padres de ambas escuelas rurales, la comisión de ambos clubes deportivos, grupo de productores que presentaron un proyecto colectivo ante un llamado del Ministerio de Ganadería Agricultura y Pesca (MGAP) en el período 2014-2015, y otros grupos no institucionalizados (Barneche et al., 2016).

1) Las SFR son asociaciones civiles, entidades con personería jurídica aprobada por el Ministerio de Educación y Cultura (MEC); tienen la particularidad de que no persiguen fines de lucro y no pueden repartir las utilidades de su gestión económica. Sus directivos son honorarios y tienen prohibido dedicarse a cuestiones ajenas a sus estatutos, como, por ejemplo, actividades políticas o religiosas. Sus asociados son principalmente productores rurales, además de personas vinculadas al quehacer agropecuario, comercial, educativo, etc., de la zona de influencia. Su adhesión es voluntaria y no es necesario el aporte de capital para ello. El ingreso y el egreso de cada asociado son libres. Las SFR se nuclean en la organización de carácter nacional Comisión Nacional de Fomento Rural (CNFR). 


\section{La experiencia de trabajo del equipo interdisciplinario}

La reconstrucción histórica del proceso con la línea de tiempo fue realizada a partir de la búsqueda y revisión de documentos de planificaciones del equipo interdisciplinario, proyecciones, planes de trabajo presentados ante el Servicio Central de Extensión y Actividades en el Medio (SCEAM) de la UdelaR, registros fotográficos, documentos generados, entre otros. $Y$ desde allí surgieron los hitos, es decir, los momentos significativos de la experiencia que denotan el proceso que realizó el equipo interdisciplinario en la integralidad.

Figura 2: Línea de tiempo de los EFI en el medio rural, 2012-2019

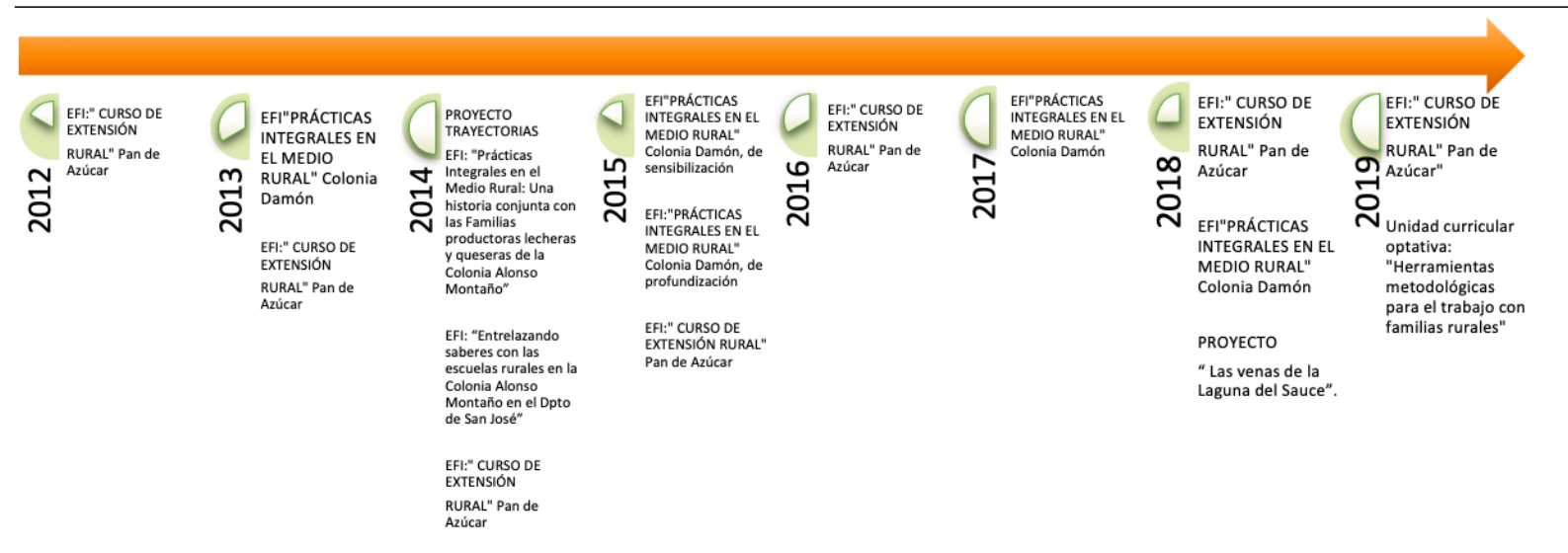

Tesis de grado

Fuente: elaboración propia.

A lo largo de estos años, los EFI se han curricularizado en los diferentes servicios universitarios implicados. En la Facultad de Veterinaria se han acreditado a partir del curso optativo Extensión rural, desde 2012. En la Facultad de Psicología, los estudiantes han podido acreditar las horas realizadas en el EFI como prácticas, reconociéndose el proceso formativo de los mismos. Con relación a los estudiantes de Nutrición, se tornó dificultoso poder implementar la curricularización de la extensión con el Plan de Estudios previo de la licenciatura, denominado Plan de Estudios de la Licenciatura en Nutrición 1998. Una de las estrategias llevadas a cabo para lograr el reconocimiento de esta experiencia fue mediante la articulación con docentes de la licenciatura, consultando las posibilidades de convalidar la experiencia en prácticas disciplinares de distintos cursos, lo cual se pudo concretar en dos casos en que los estudiantes realizaron un trabajo complementario a partir de la experiencia con las familias de la Colonia AM. Cabe señalar que no fue hasta la implementación del nuevo Plan de Estudios de la Licenciatura en Nutrición (Plan 2014) que los EFI pudieron ser acreditados para los estudiantes de Nutrición, ya que fueron enmarcados como una unidad curricular optativa.

La Escuela de Nutrición, siguiendo los lineamientos de la Segunda Reforma Universitaria, luego de un proceso de construcción colectiva que incluyó una evaluación institucional (2008), en el año 2014 aprobó el Plan de Estudios de la Licenciatura en Nutrición, el que se comenzó a implementar en marzo de 2018. Dicho plan pretende la promoción de una con- 
cepción integral de la enseñanza, busca desarrollar procesos de aprendizaje orientados al logro de la más amplia y rica formación de los estudiantes, a la comprensión crítica y creativa del conocimiento, de la sociedad y sus problemas, y consecuentemente a un solvente y ético desempeño profesional. La articulación de las funciones de enseñanza, investigación y extensión, apuntando especialmente a la curricularización de la extensión, aparecen como una oportunidad para lograr los cometidos planteados, discusión que fue parte central en el diseño de este plan de estudios y que entendimos necesaria dar y estimular. La flexibilidad curricular, por medio de actividades opcionales y electivas, permitió entonces presentar desde el año 2019 el curso optativo interservicio dentro de la oferta académica para los estudiantes de Nutrición.

Los grupos de trabajo en cada uno de los EFI desarrollados fueron conformados por estudiantes de los tres servicios a través de llamados abiertos comunicados por las vías formales institucionales y por las redes sociales y cartelería. Cabe señalar que transitaron por estas propuestas alrededor de 150 estudiantes de la Licenciatura en Nutrición, un número similar de estudiantes de Psicología y más de 300 de Veterinaria. También participaron estudiantes de otros servicios, como Comunicación, Trabajo Social, Licenciatura en Desarrollo y Ciencias. Para ingresar a los EFI se entiende que no es necesario establecer materias previas, ya que todos y todas cuentan con experiencias que pueden aportar al proceso, que lo trabajado trasciende el momento concreto de la formación, que se van gestando aprendizajes que luego serán resignificados. Por cada una de las propuestas el equipo estableció un cupo máximo de aproximadamente 40 estudiantes, contemplando aspectos pedagógicos y de logística en cuanto al trabajo en territorio.

Estos EFI tienen como objetivo que los estudiantes se introduzcan en los aspectos teórico-metodológicos de la extensión, se acerquen a la realidad concreta de las familias productoras y de las instituciones y redes de la zona de influencia.

Asimismo, el equipo interdisciplinario procura contribuir a través de los EFI al proceso de consolidación de los colectivos rurales para la búsqueda de soluciones conjuntas a las demandas detectadas por la comunidad, para la mejora de la calidad de vida.

Es relevante destacar que en los últimos años se ha dado un conflicto entre dos modelos prácticamente antagónicos: el agronegocio y la agricultura familiar. Se ha dado un deterioro de la identidad del productor familiar como tal, incorporándose muchas veces al modelo tecnológico imperante (Barneche et al., 2016). En contraposición el acceso a políticas públicas de desarrollo rural, pensadas para favorecer la sustentabilidad de la producción familiar impulsadas por la Dirección General de Desarrollo Rural (DGDR), ${ }^{2}$ han sido una posibilidad para disminuir el impacto de la lógica del SAA global. Remitiendo a este como:

"una serie de actividades que involucran la producción, el procesamiento industrial, la comercialización y la distribución final de los alimentos, orientados tanto al mercado interno como a las exportaciones; (...) —desde la semilla (o la genética animal) a la mesa del consumidor-, así como el conjunto de agentes económicos y sociales y las relaciones de éstos en cada una de las etapas del proceso de transformación, articulando entre sí y/o con agentes que participan en otras etapas del sistema. Asociados al SAA se 
encuentran los sistemas de soporte o de infraestructura; los sistemas educativo y científico tecnológico, los financiamientos, los mecanismos de comercialización y regulación". (Teubal y Rodríguez, 2002, en Carballo, 2018, p. 22)

De esta manera se identifican dos paradigmas en tensión puesto que, por un lado, se promueve continuar con la lógica del SAA global imperante, siendo un país dependiente del mercado externo, con un rol de productor y exportador de materias primas, y por el otro, alineados con las políticas de protección de la producción familiar, los EFI intentan acompañar los procesos colectivos de las familias rurales para estimular el desarrollo y fortalecimiento del SAA local mediante el fomento y la revalorización de la producción y la comercialización de alimentos para el consumo a nivel local.

Como estrategia de trabajo se desarrollan los "vivenciales", que son jornadas de convivencia a nivel predial donde se da el intercambio entre estudiantes y las familias y a nivel colectivo. Las demandas son construidas involucrando a las familias productoras, en conjunto con el equipo docente y los estudiantes, así como en la implementación y evaluación de las actividades. Es clave también realizar coordinaciones con las instituciones que se encuentran en el territorio de modo de integrarlas en la propuesta, generar o fortalecer redes y mejorar el acceso a las políticas en el medio rural.

El equipo docente entiende que para establecer un proceso formativo con los estudiantes es necesario la realización de un espacio de enseñanza-aprendizaje con el fin de promover el trabajo en grupos interdisciplinarios en pro del diálogo, derribando las barreras disciplinares, reflexionando sobre el quehacer de las instancias en terreno, fomentando el involucramiento y la participación activa de los estudiantes. Además, se busca que los estudiantes pongan en práctica algunas herramientas e instrumentos teórico-conceptuales en el incipiente ejercicio del rol, así como sensibilizar en una actitud de trabajo, en la que sean bases el diálogo y la construcción de saberes con todos los actores implicados, y facilitar la reflexión sobre diversas problemáticas que afectan a una comunidad rural. Adhiriendo a la definición de Rectorado UdelaR (2010), "las tareas se generan y operan en terreno, partiendo de los problemas que la sociedad tiene, intentando junto con ella encontrar alternativas, entonces el acto educativo se re-configura y amplía" (p. 15).

Cabe destacar que se trabajan contenidos teóricos para retroalimentar las prácticas que se abordan en orden, formato y metodología distintos según los intereses de los participantes, donde se destaca la demanda que se construye. Es así que se abordan los procesos de alimentación y nutrición estudiando los entornos reales de las poblaciones, con una visión histórica, contemporánea y prospectiva, que considera los diferentes determinantes políticos, sociales, económicos, culturales, ambientales y éticos que inciden en el bienestar nutricional de la población. El estudio constante del contexto político, económico, social y cultural, vinculándolo a las políticas públicas, la investigación y la formación profesional.

\section{Hitos de la experiencia EFI en el medio rural}

Los hitos, entendidos como los momentos significativos de la experiencia, fueron rescatados a través de la nube de palabras (Figura 3). La misma fue realizada utilizando la plataforma Mentimeter, a partir del ingreso de palabras que representaran la consigna "Momentos sig- 
nificativos de los EFI 2012-2019". Esta técnica recoge a modo de lluvia de ideas los hitos percibidos de forma subjetiva, definiéndose en función del objetivo de este trabajo. Entre ambas metodologías es posible contextualizar los hitos al identificar las fechas en que se dieron, quiénes participaron $y$, sintéticamente, qué fue lo que sucedió. Para contemplar en cada uno las distintas dimensiones de la acción humana: "el hacer, el sentir y el pensar" (Berrutti, Cabo, Dabezies, 2012, p. 82), se elaboró una encuesta en formato Google docs con la finalidad de rescatar la experiencia de los estudiantes de Nutrición que transitaron por el EFI.

Figura 3: Nube de palabras de los hitos EFI 2012-2019

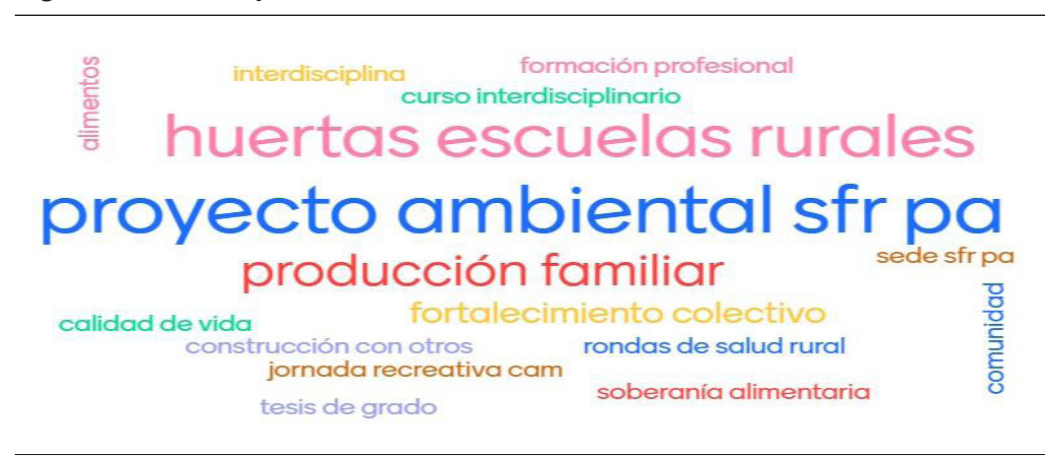

Fuente: elaboración propia.

\section{El proyecto de conservación ambiental de la SFR PA}

La SFR PA, desde hace unos años, viene trabajando en la construcción de un plan estratégico que se orienta a posicionar a la organización como referente del territorio, en la búsqueda de aportar al desarrollo rural sustentable. Como punto de partida se realizó un diagnóstico y se delinearon las estrategias contemplando tres dimensiones: social/cultural, ambiental y económica. En el mismo se destaca la producción familiar como sector central que aporta a la cultura rural y a la sociedad en su rol de productora de alimentos inocuos, sumado a un modo en particular de producción. También es relevante la preocupación por los procesos y transformaciones en el medio rural que afecta, modifica el ambiente y degrada algunas de las zonas, especialmente con la contaminación de las fuentes de agua. En este marco, en el año 2017 se consolidó este trabajo en un proyecto que la SFR PA presentó ante el DGDR, Ilamado "Las venas de la Laguna del Sauce". El EFI se centró en contribuir con los objetivos planteados en el proyecto en cuanto a desarrollar acciones de sensibilización, comunicación y capacitación orientado a un modelo de desarrollo sustentable y amigable con sentido de pertenencia del medio ambiente. También se colaboró con la implementación de una estrategia de relevamiento y diagnóstico de situación a nivel territorial y predial. Los dispositivos utilizados a nivel predial y colectivo que contribuyeron fueron: visitas prediales con modalidad de vivencial, mapeo predial, y taller colectivo con las familias socias con la construcción del árbol de problemas. En las instancias colectivas participaron las instituciones vinculadas al medio rural.

Respecto de los aprendizajes, una estudiante de Nutrición que transitó por esta experiencia destacó, en el formulario de encuestas realizado por la plataforma Google docs, la posibilidad de hacer visible "la relación entre política, ambiente, producción y abastecimiento de los alimentos". Durante todo el proceso de trabajo del EFI se puso en relieve 
el eje de la sustentabilidad en sentido amplio, contemplando el cuidado de los recursos naturales, entendiéndose no solo como elemento clave para una producción sustentable de alimentos sino también para el desarrollo de una adecuada calidad de vida. Como también la necesidad del análisis de la influencia del funcionamiento del SAA en la situación alimentaria y nutricional de las poblaciones.

Los dispositivos utilizados favorecieron la participación, la reflexión y el aprendizaje sobre la contaminación de la cuenca, sus causas y consecuencias, y para hacer visible que todos y todas somos parte del problema y de la solución del mismo. De esta manera, el EFI aportó al diálogo de saberes entre el equipo interdisciplinario de la UdelaR y la SFR PA, así como al intercambio con otras instituciones de la zona implicadas en la temática del cuidado de la laguna.

\section{Huertas con las escuelas rurales}

Las escuelas rurales como instituciones presentes en los territorios aparecen como una oportunidad para el desarrollo de acciones a nivel comunitario y familiar.

En la Colonia AM, como parte del trabajo conjunto con las escuelas rurales, en el año 2013 fue realizado el EFI "Entrelazando saberes con las escuelas rurales en la Colonia Alonso Montaño en el Dpto. de San José", que tenía como objetivos: contribuir a la revalorización del centro educativo como productor de conocimientos y articulador con la comunidad rural en las escuelas rurales de la Colonia AM y acercar a los estudiantes al trabajo en extensión rural, con la comunidad y en interdisciplina, fomentando la integralidad de los saberes.

Se trabajó en ambas escuelas de la Colonia, construyendo en conjunto las demandas. Fueron realizados talleres en torno a dos temas: sexualidad y alimentación. Respecto de esto último, se trabajó específicamente en torno a hábitos de alimentación saludables, la obtención de leche y la elaboración de queso en el marco del sistema agroalimentario local. Además, en la Escuela $N^{\circ} 92$ se hizo la reconstrucción de la huerta escolar y se apoyó la preparación y ejecución de los festejos de los 50 años de la misma. Al respecto, se rescata la experiencia de una estudiante que relata que el EFI le aportó a su formación "en educación" la realización de talleres, así como "el diagnóstico, la planificación y la ejecución del trabajar en equipos multidisciplinarios y la comunicación con otros".

Posteriormente, en 2017, en el marco del EFI "Prácticas Integrales en el Medio Rural", se trabajó en dos grupos interdisciplinarios en forma simultánea con ambas escuelas y con las familias. Cada grupo planificó la intervención en diálogo con los actores de la comunidad (maestras, familias y referentes). Se planificó, ejecutó y evaluó en conjunto una serie de talleres que implicaron distintos momentos en torno a la huerta orgánica, en los cuales se abordaron los siguientes aspectos: construcción del calendario de siembra y cosecha, etapas de la elaboración de la huerta escolar, características de los vegetales y las frutas (ciclos, aspectos sensoriales, aporte nutricional), elaboración de un semillero para la huerta escolar, remedios caseros para evitar plagas en la huerta orgánica, recuperación de la huerta (delimitado y cercado, elaboración de canteros, acondicionamiento de la tierra, elaboración de compost, realización de plantines y siembra de forma directa en canteros, realización de carteles indicativos y espantapájaros). Se destaca la importancia de la participación como estudiantes en la fase de producción de alimentos en el SAA para comprender su complejidad, lo cual posibilitó una visión integral del mismo. 
Con relación al territorio PA, en 2016 se trabajó en dos escuelas rurales, № 31 y № 72 , en el marco del EFI "Extensión rural en Pan de Azúcar". El trabajo surgió como una demanda de la SFR PA con el objetivo de generar un espacio de integración entre la misma y las instituciones locales, partiendo de la importancia que tiene el trabajo en conjunto para el fortalecimiento de la zona. La tarea se llevó a cabo a partir de la conmemoración del festival "Fiesta del Árbol", donde los estudiantes, acompañados por socios de la SFR, plantaron un árbol de frutos autóctonos (Arazá), degustaron mermeladas elaboradas por las socias y realizaron un taller donde se abordó la temática de árboles autóctonos (usos, cuidados necesarios, relevancia para el ecosistema). El trabajo en las escuelas fue implementado con la SFR PA, que coordinó con las escuelas, aportó el árbol y las mermeladas, mientras los estudiantes asumieron la responsabilidad de planificar la actividad y preparar el taller (Vasconcellos et al., 2018).

Dichas instancias son valoradas por el equipo contemplando a las escuelas como espacios propicios y facilitadores del intercambio, así como promotoras de la participación de distintos actores, desde las propias familias, las maestras, los niños y niñas, la universidad a través de los estudiantes y docentes y el colectivo de familias rurales.

A partir de estas experiencia se potencia el aprendizaje de competencias genéricas y específicas que hacen al licenciado en nutrición, como son desarrollar habilidades y actitudes que faciliten la comprensión de los problemas alimentarios y nutricionales y sus factores condicionantes; planificar, ejecutar y evaluar planes y programas alimentario-nutricionales y educativos; intervenir en proyectos y programas interdisciplinarios de mejora de la calidad de vida y desarrollo social y productivo, entre otros aspectos señalados en el Plan de Estudios (Escuela de Nutrición, UdelaR, 2014).

\section{Rondas de salud rural}

Otro de los momentos significativos en el marco del EFI durante el año 2015, fueron las Rondas de Salud realizadas en las escuelas de la Colonia AM. Las mismas son actividades de sensibilización y atención en el cuidado de la salud de niños y niñas participantes de las escuelas públicas de las regiones rurales del departamento de San José, sus familias y la población rural en general. Son jornadas de promoción y prevención de salud organizadas por Ministerio de Salud Pública, Comisión Zoonosis, Administración Nacional de Educación Pública, entre otras instituciones vinculadas al medio rural y que tienen mucha importancia para la zona. Las Rondas de Salud son valoradas muy positivamente por las escuelas y las familias, acercando una variedad de instituciones a la zona, las cuales son de difícil acceso para las familias. Algunas de las actividades de las Rondas fueron: atención médica, odontología, enfermería, vacunación, realización del Papanicolaou así como talleres de alimentación saludable y manejo de agroquímicos. Además se efectuaron actividades para el manejo responsable de mascotas y de promoción y capacitación que incluyeron las temáticas como: salud ocupacional, salud sexual y reproductiva, adicciones, información sobre beneficios y uso de servicios públicos, y también actividades recreativas y de educación física para niños.

Para la primera ronda de salud, la maestra convocó al equipo docente a participar con los estudiantes en la difusión del evento, lo cual se llevó a cabo a través de visitas prediales acercando la información sobre la realización de la Ronda y los servicios que ofrecía, intercambiando con las familias la importancia de contar con los mismos. En la segunda Ronda 
se produjo un cambio en la participación del equipo, que se incorporó a las instituciones que trabajaron en la preparación de dicha Ronda. Se planificó conjuntamente con estudiantes, docentes, familias y referentes de las instituciones la realización de talleres y actividades a realizar. Este ámbito interinstitucional permitió reflexionar con los estudiantes a la luz de la experiencia sobre las complejidades del trabajo en este nivel (coordinación, objetivos, formas de trabajo de las instituciones, entre otros aspectos), así como identificar características que hacen a la vulnerabilidad de las familias rurales en cuanto al acceso a servicios que condicionan el completo cumplimiento de los derechos humanos.

\section{Tesis de grado}

En el marco de los EFI, estudiantes de Nutrición que participaron de los mismos presentaron interés de profundizar en algunas de las temáticas surgidas en la experiencia. Uno de los estudiantes encuestados manifestó:

"La extensión rural nos acerca más a la parte pedagógica que adquirimos en las diferentes materias (...), logrando así ver los problemas en territorio, poniendo en práctica la diferencia alimentaria del interior con el centro urbano. Dándonos la posibilidad de poder desarrollar práctica, hacer tareas de investigación y vincular nuestro conocimiento con otras disciplinas". (Estudiante EFI, 2018)

En tanto, hubo tesis de grado de la Licenciatura en Nutrición que fueron llevadas a cabo en esos años, las que se presentan en la Cuadro 1.

Cuadro 1: Tesis de grado realizadas desde 2012 a 2019

\begin{tabular}{|c|c|}
\hline Año & Título \\
\hline 2018-2019 & $\begin{array}{l}\text { Prácticas alimentarias de los productores familiares rurales socios de la Sociedad de fomento rural } \\
\text { de Pan de Azúcar }\end{array}$ \\
\hline 2018 & Prácticas alimentarias de las familias que viven en la Colonia A. Montaño, inmueble Damón \\
\hline 2014 & $\begin{array}{l}\text { Estrategias alimentarias de las familias productoras queseras y lecheras de la Colonia A. Montaño, } \\
\text { inmueble Damón }\end{array}$ \\
\hline 2014 & $\begin{array}{l}\text { Condicionantes de la sustentabilidad de la producción lechera y quesera familiar de la Colonia A. } \\
\text { Montaño, inmueble Damón }\end{array}$ \\
\hline 2012-2013 & Seguridad alimentaria y producción familiar en la Sociedad de Fomento Rural de Pan de Azúcar \\
\hline
\end{tabular}

Fuente: elaboración propia.

La realización de tesis de grado fue la estrategia identificada para curricularizar los aprendizajes generados en los EFI en los estudiantes. A su vez, reorientar las líneas de investigación y los procesos de enseñanza desde la función de extensión de modo de generar compromiso universitario con la sociedad en la resolución de sus problemas alimentarios nutricionales más significativos. Estos trabajos fueron hechos por grupos de dos a cuatro estudiantes; las docentes tutoras fueron las integrantes del equipo interdisciplinario pertenecientes a la Escuela de Nutrición. Los temas abordados fueron desde los marcos conceptuales del DAA, la Seguridad Alimentaria y Nutricional (SAN), la Soberanía Alimentaria $(\mathrm{SbA})$, siendo estos conceptos necesarios para comprender la complejidad de los problemas 
alimentario-nutricionales a nivel poblacional, dado que amplían la mirada sobre la situación alimentaria y nutricional, contribuyen a visualizar los factores condicionantes que influyen en el estado o situación de la misma, y permiten acciones integrales que aborden los problemas identificados para su resolución.

\section{Trabajo con las familias y el colectivo rural}

El equipo interdisciplinario, desde el inicio de trabajo con los territorios implicados, entendió a las familias productoras y a los colectivos rurales como parte fundamental para la construcción de los espacios y la comprensión de las realidades rurales, siendo un eje del trabajo la reconstrucción de la identidad del ser familia productora y de los colectivos rurales.

Es de relevancia identificar aquellos aspectos que caracterizan a los productores agropecuarios familiares. Según las Resoluciones del MGAP 219/14 y 387/14, son personas físicas que gestionan directamente una explotación agropecuaria y/o realizan una actividad productiva agraria que no requiere el uso directo de la tierra. Estas personas deben, en conjunto con su familia, efectuar la explotación contratando o no un máximo de dos asalariados no familiares permanentes o su equivalente en jornales, explotar en total hasta 500 hectáreas (Coneat 100) bajo cualquier forma de tenencia, residir en la explotación o a una distancia menor a 50 kilómetros de la misma, con ingresos familiares nominales extraprediales que sean inferiores o iguales a 14 BPC (Bases de Prestación Contributiva) de promedio mensual.

Más allá de estos aspectos normativos, como equipo interdisciplinario acordamos con Fernández y Carámbula (2012) que la producción familiar se encuentra sustentada por el trabajo familiar, el uso de la tierra y la articulación con los mercados. También es importante resaltar, como indica Piñeiro (1994), que se pueden identificar a dos subcategorías: el productor familiar capitalizado y el pluriactivo, diferenciándose por su capital. Los segundos poseen poca tierra o capital y salen a trabajar fuera de su establecimiento una parte del año para incrementar los ingresos del hogar (p. 11).

Es necesario incluir una dimensión que el equipo entiende relevante: el ser productor y productora familiar implica un modo de vida caracterizado por una vinculación particular con la naturaleza, con tiempos y costumbres propios. El trabajo con estos actores se basa en la modalidad denominada "vivencial" como el principal dispositivo, que se trata de un espacio en donde

"las familias productoras se transforman en socios pedagógicos de los estudiantes universitarios, generando entre ellos un vínculo imprescindible para la construcción de un proceso de extensión educativo, crítico y transformador". (Sapriza et al., 2016)

La familia y los estudiantes

"se encuentran en el predio, compartiendo el espacio cotidiano y la rutina de las familias, las historias personales, develando las prácticas de producción y elaboración de los productos, poniendo en juego visiblemente el diálogo de saberes". (Sapriza et al., 2016)

Además, a lo largo del tiempo se han puesto en práctica otras metodologías que complementan a las vivenciales, como son el mapeo vincular (social y productivo) de cada familia, árbol de los miedos y sueños familiares, historias de vida, talleres, diario de vida de construcción colectiva. 
Cabe mencionar que también fue realizado el análisis de la inocuidad de los quesos producidos para determinar la presencia de aflatoxinas, actividad efectuada en coordinación con el área de toxicología de Facultad de Veterinaria, cuyos resultados fueron discutidos en modalidad de taller con las familias productoras de la Colonia.

Entendemos que a partir de los diferentes dispositivos se logró reflexionar en conjunto con los estudiantes sobre la concepción de producción familiar y su rol en el SAA. En este sentido, conocer la brecha (distancia geográfica, actividades y relaciones) que existen entre la producción y el consumo de alimentos es clave para analizar el cumplimiento de la función de la alimentación humana en una sociedad, por lo que es primordial tener en cuenta que los procesos de producción, transformación, distribución y consumo de alimentos terminan reconfigurando las relaciones sociales y económicas de una sociedad.

\section{Reflexiones}

Los EFI en el medio rural implementados por el equipo constituyeron un importante antecedente para la concreción del Plan de Estudio de la Licenciatura en Nutrición aprobado en 2014 y comenzado en 2018. Los mismos son un dispositivo invaluable para el desarrollo de las competencias que en él se promueven, fundamentalmente las denominadas competencias genéricas. En este sentido, se destaca que durante todo el proceso de trabajo se reflexionó sobre las características propias de la formación disciplinar, fortaleciéndose y consolidándose en el encuentro con otras disciplinas y saberes, concretando el trabajo interdisciplinario que dicho plan de estudios plantea. En la misma línea, las metodologías utilizadas promovieron el pensamiento crítico y propositivo y la autonomía en la toma de decisiones en cuanto a las actividades relacionadas con los EFI. Esta propuesta también posibilita un rol activo de los estudiantes, quienes revalorizan el trabajo grupal e interdisciplinario a través de la práctica con la comunidad, la discusión y reflexión permanente, lo cual les permite tomar decisiones sobre el proceso de trabajo con los territorios, haciéndolos partícipes de su propio recorrido, en donde el diálogo habilita un encuentro real, en donde los diferentes mundos, historias y experiencias de los sujetos confluyen, se descubren y se transforman, destacando las experiencias previas de todos los involucrados y trayectorias personales para ponerlas en juego y construir en conjunto nuevos aprendizajes y saberes.

La realización de tesis de grado favoreció la articulación de todas las funciones universitarias: la enseñanza, la extensión y la investigación. Las mismas surgieron por el interés de los estudiantes en profundizar los conocimientos vinculados a la disciplina en este marco, lo cual significó un enriquecimiento del proceso formativo de los mismos. Esto se encuentra en consonancia con las competencias específicas promovidas por el plan de estudios sobre la intervención en proyectos y programas interdisciplinarios de mejora de la calidad de vida y desarrollo social y productivo, en investigaciones que respondan a problemas de la práctica profesional, entre otras. Esta experiencia da cuenta de que para el abordaje de la salud en forma integral es necesario construir una interdisciplina con servicios del área salud y agraria, por mencionar solo dos. Se destaca que el EFI logra, tal como plantea el Plan de Estudios 2014, la revalorización del abordaje interdisciplinario de los problemas alimentario-nutricionales, basándose en la tridimensionalidad del concepto de la ciencia de la nutrición, por lo que toma en consideración al individuo en su contexto, es decir, no solo 
desde una dimensión biológica sino también como un ser psicológico, cultural y social en constante relación con el medio (Cannon, 2005), buscando estimular el abordaje multicausal de dichos problemas.

Comprender el SAA, su dinámica, y revalorizar la fase de producción como parte del mismo es clave para que el licenciado en Nutrición pueda analizar la situación alimentaria y nutricional en su complejidad. Habitualmente, los profesionales de la nutrición suelen centrarse en el consumo de alimentos y en el estado nutricional, en tanto que la práctica con el medio rural permite conectarse con todas las fases del SAA, utilizando como base para su análisis los marcos conceptuales de SAN y SbA, los cuales hacen posible una visión integral, que contemple la multidimensionalidad de los factores que inciden el ejercicio del DAA.

A su vez, el trabajo en comunidad como parte del desempeño profesional, en conjunto con los actores sociales, contribuye a dar explicación a los problemas alimentarios y nutricionales y a extender la mirada a los determinantes políticos, socioeconómicos y medioambientales contemplando la visión de la propia comunidad.

\section{Referencias bibliográficas}

Berrutti, L.; Cabo, M.; Dabezies, M. (2014). Apuntes para la acción III - Sistematización de experiencias de extensión universitaria. Extensión Libros. Comisión Sectorial de Extensión y Actividades en el Medio (CSEAM). Barneche, M.; Cauci, A.; Sapriza, D.; Ferrari, N.; Gandolfo, A.; Gandolfo B. (...); Risso F. (2016). Lo grupal en la Colonia Damón: develando los colectivos que viven y construyen la Colonia. http://extension.unicen.edu.ar/ jem/subir/uploads/1526_2016.pdf

Cannon, G.; Leitzmann, C. (2005). The new nutrition science project. Public Health Nutr.

Carballo, C. (2018). Soberanía alimentaria y desarrollo: caminos y horizontes en Argentina. Cuadernos para la soberanía alimentaria; \#2. Mónadanomada.

Fernández, E.; Carámbula, M. (2012). Territorios en disputa: la producción familiar en el este uruguayo. https://www.researchgate.net/publication/314787417_Territorios_en_disputa_la_produccion_familiar_en_ el_este_uruguayo

Manchado, R.; Tamames, S.; López, M.; Mohedano, L.; D’Agostino, M. \& Veiga de Cabo, J. (2009). Revisiones Sistemáticas Exploratorias. Medicina y Seguridad de/ Trabajo, 55(216), 12-19. http://scielo.isciii.es/scielo. php?script=sci_arttext\&pid=S0465-546X2009000300002\&lng=es\&tlng=pt

MGAP (s.f.). Ajustes en la definición de productor familiar permitirá un mayor acceso a políticas diferenciadas. https://www.gub.uy/ministerio-ganaderia-agricultura-pesca/comunicacion/publicaciones/produccion-familiar-integral-sustentable-documentos-consulta

Piñeiro, P. (1994). Tipos sociales agrarios y nacionalidad productiva: un ensayo de interpretación. Departamento de Ciencias sociales. Facultad de Agronomía. UdelaR.

Plan de Estudios (2014). Escuela de Nutrición. UdelaR.

Rectorado UdelaR (2010). Hacia la reforma universitaria \#10. La extensión en la renovación de la enseñanza: Espacios de Formación Integral. http://www.extension.udelar.edu.uy/wp-content/uploads/2016/12/08_ Hacia-la-reforma-universitaria_-la-extensio\%CC\%81n-en-la-renovacio\%CC\%81n-de-la-ensen\%CC\%83anza.pdf

Sapriza, D.; Cauci, A.; Barneche, M.; Ferrari, N.; Gandolfo, A.; Gandolfo, B. (...); Risso, F. (2016). Los vivenciales habilitadores del trabajo en la Colonia Alonso Montaño. http://extension.unicen.edu.ar/jem/subir/ uploads/1527_2016.pdf 
Vasconcellos, M.; Fraga, S.; Méndez, S.; Ferrari N.; Gandolfo, B.; Sapriza, D. (...); Muniz, F. (2018). Sobre nuestros pasos: cosechando experiencias, a dos años de un Espacio de Formación Integral. Poster y Ponencia. Jornadas Nacionales de Extensión organizadas por la Comisión Sectorial de Extensión de la Universidad de la República. Departamento de Maldonado, Uruguay. https://www.colibri.udelar.edu.uy/jspui/handle/20.500.12008/20583 